\title{
Ultraviolet Radiation (UVR) and Risk of Developing Multiple Sclerosis (MS)
}

Can. J. Neurol. Sci. 2011; 38: 6-7

One of the most widely accepted epidemiological features of multiple sclerosis (MS) is its tendency to increase as latitude increases, in both directions from the equator. The worldwide distribution of MS may be an indirect reflection of some cause which varies by latitude, the most obvious environmental factor being climate. The possible role of ultraviolet radiation (UVR) in MS etiology was first proposed in $1960^{1}$ and has recently received increasing attention. This issue of the Canadian Journal of Neurological Sciences includes a paper by Sloka et $\mathrm{al}^{2}$ which correlates MS prevalence and UVR globally, along with latitude and other factors that might contribute to the geographic distribution of MS. While this study furthers our understanding of the relative bearing which UVR may have on MS distribution, it also highlights some of the difficulties inherent in examining UVR's impact.

Sloka et al observed a statistically significant negative correlation between UVR and MS prevalence rates worldwide. ${ }^{2}$ The use of prevalence rates to assess whether low UVR exposure is a risk factor for developing MS is not unusual, because incidence rates are less available and more susceptible to measurement error; however it is problematic. Prevalence rates can lack biological relevance since MS patients living in a particular region may have been elsewhere when their disease began. The few studies using a measure of incidence have targeted various age periods according to different theories about when MS begins, in particular that some important cause occurs before age 15 based on migrant studies ${ }^{3}$. An earlier paper by Sloka et $\mathrm{al}^{4}$ reported a statistically significant negative correlation between UVR and MS patients' place of residence in Newfoundland during their first year of life, a smaller correlation for residence over the first ten years, and no correlation with residence at symptom onset. Beretich and Beretich ${ }^{5}$ suggest that the negative correlation between UVR and statewide MS prevalence based on US veterans' data implicates UVR in incidence because subjects were predominantly born and lived in the state where they were inducted around age 18 . Staples et $\mathrm{al}^{6}$ found a negative correlation between residential UVR in the first trimester of pregnancy and risk of having an infant who later developed MS, in addition to a negative correlation between UVR and region of birth among Australian MS patients. The various age periods used in these studies complicates an assessment of the relationship between UVR and incidence. It remains unclear whether UVR is involved in either the initiation or clinical manifestation of MS.

Sloka et al conclude that low UVR exposure is a risk factor for the development of MS which outweighs other factors in their study, including latitude, by at least 20 -fold. ${ }^{2}$ The data presented only illustrates this 20 -fold risk through p-values from single variate regression analyses. P-values indicate the likelihood that a relationship is observed by chance rather than quantifying effect size. The coefficients in Figure 2 of the paper, which might more reasonably be considered a measure of the degree of relationships, indicate that UVR has only a slightly stronger correlation with MS prevalence at 0.37 than with latitude at 0.30 . The emphasis on statistically significant $p$-values rather than meaningful effect size can be found in other UVR studies; and in fact where relationships have been found between UVR and MS distribution, correlation coefficients or other risk statistics such as odds ratios (ORs) tend to be small ${ }^{4-6,7}$. Most reported ORs for example are less than 2:1 for low UVR exposure, too small to reach the threshold beyond which an elevated OR is probably not due to bias ${ }^{8}$. Considering the typically small effect sizes which have been found, Sloka et al's recommendation that UVR could be used by health ministries for estimating their MS populations and allocating resources might be tempered by recognition that other factors related to prevalence could confound any such approach. The lower than expected MS prevalence in Scandinavia considering available UVR might illustrate this need for caution.

Added to concern about effect size is the concern that measurement of both MS prevalence and UVR varies among studies including size of measurement error, as does the measurement of other possible explanatory factors. Sloka et al's use of multiple regression to assess the relative bearing of UVR and other factors on MS distribution moves beyond several studies which have addressed UVR alone or UVR controlling for latitude. However it also demonstrates that the way in which variables are measured or entered into a multiple regression analysis can influence whether or what part of the variance in MS distribution they explain. For example, when latitude and longitude were entered separately all other factors fell out of the model, but when latitude and longitude were combined into a variable representing distance from northern Europe, smoking and accuracy of methods used to measure prevalence entered the model as negatively significant. The reason for changes in these configurations is not always readily apparent.

All this aside, the statistically significant negative correlation between UVR and MS prevalence observed by Sloka et al is consistent with the findings of other recent descriptive studies which have explored the relationship between UVR and MS prevalence ${ }^{5,7}$. However, because they do not examine exposure to UVR at the individual level, that is, exposure of MS patients versus controls retrospectively or via comparison groups prospectively, all such studies are limited in their ability to establish cause. As Sloka et al note, many factors can influence exposure to UVR regardless of its level in the atmosphere, including amount of time people spend outdoors, clothing worn, sunscreen use and skin pigmentation. ${ }^{2} \mathrm{~A}$ few case-control studies have been conducted addressing the possibility that low UVR is a risk factor for developing MS, but the results of these studies are less consistent. Norman, Cook and Dowling 9 found that low UVR exposure was not a statistically significant MS risk factor, 
when controlling for latitude. Other studies have found no difference between cases and controls in terms of outdoor occupation or recreational activities ${ }^{10,11}$. More recently Kampman et $\mathrm{al}^{12}$ found that Norwegian MS patients reported lower outdoor summertime activities than controls, most pronounced between ages 16-20; and van der Mei et $\mathrm{al}^{13}$ found that MS patients reported spending fewer hours per day outdoors on weekends and holidays year round than controls, particularly between ages 6-15. Case-control studies have their own limitations such as recall bias where subject report is used. Even objective measures, such as sun damage to skin which van der Mei et al $^{13}$ used to supplement subject report, can be compromised since timing of damage prior to onset age could not be established. Nevertheless it is probably time to encourage more, soundly designed case-control studies to clarify the relationship between UVR and risk of developing MS.

Several mechanisms have been suggested through which UVR may play a role in the development of MS. Sloka et al emphasize the possibility that UVR could act through alterations in Vitamin $\mathrm{D}_{3}$ which displays immunomodulatory properties in humans. For example, low UVR could have a negative impact on the immune system through deficient Vitamin $\mathrm{D}_{3}$ production, which in turn might increase susceptibility to attack by some causal virus like Epstein-Barr ${ }^{14}$. UVR could act through other possible mechanisms however which should not be overlooked, such as changes in level of melatonin which also has immunomodulatory properties ${ }^{15}$. Or UVR may have a direct immuno-suppressive effect, for example, by reducing the ratio of helper to suppressor T-cells ${ }^{16}$. On the other hand, latitude's independent contribution to MS prevalence which Sloka et al observed suggests that both latitude and UVR may be proxies for a third factor which is actually causal, such as ionizing cosmic radiation which might induce oxidative myelin damage in MS patients ${ }^{17}$. Some epidemiological observations do not fit a causal role for UVR in the development of MS and remain to be resolved, among them: that the incidence of MS appears to be unchanged or increasing; but, if high UVR is protective against MS, then incidence should decline with the increase in UVR which is part of global warming ${ }^{18}$.

Sharon Warren, Kenneth G. Warren, Karen V.L. Turpin University of Alberta Edmonton, Alberta, Canada

\section{REFERENCES}

1. Acheson ED, Bachrach CA, Wright FM. Some comments on the relationship of the distribution of multiple sclerosis to latitude, solar radiation and other variables. Acta Psychiatr Scand. 1960; 35 Suppl 147:132-47.

2. Sloka S, Silva C, Pryse-Phillips W, Patten S, Metz L, Wee Yong V. A quantitative analysis of suspected environmental causes of MS. Can J Neurol Sci. 2011;38(1):98-105.

3. Wolfson C, Wolfson DB, Zielinski JM. On the estimation of the latent period of multiple sclerosis. Neuroepidemiology. 1989;8: 239-48.

4. Sloka JS, Pryse-Phillips WEM, Stefanelli M. The geospatial relationship between ultraviolet radiation and multiple sclerosis in Newfoundland. Can J Neurol Sci. 2008;35(1):69-74.

5. Beretich BD, Beretich TM. Explaining multiple sclerosis by ultraviolet exposure: a geospatial analysis. Multiple Sclerosis. 2009;15:891-8.

6. Staples J, Ponsonby A-L, Lim L. Low maternal exposure to ultraviolet radiation in pregnancy, month of birth, and risk of multiple sclerosis in offspring: longitudinal analysis. BMJ. 2010;340.c1640.

7. van der Mei IAF, Ponsonby A-L, Blizzard L, Dwyer T. Regional variation in multiple sclerosis prevalence in Australia and its association with ambient ultraviolet radiation. Neuroepidemiology. 2001;20:168-74.

8. Lilienfeld AM, Lilienfeld DE. Foundations of epidemiology. 2nd ed. Oxford University Press; Oxford, United Kingdom: 1980.

9. Norman JE Jr, Cook SD, Dowling PC. Pilot survey of household pets among veterans with multiple sclerosis and age-matched controls. Arch Neurol. 1983;40:213-4.

10. Leibowitz U, Alter M. Multiple sclerosis: clues to its cause. North Holland, Amsterdam, Netherlands, 1978.

11. Warren S, Warren KG. Multiple sclerosis and diabetes mellitus: further evidence of a relationship. Can J Neurol Sci. 1982;9: 415-9.

12. Kampman MT, Wilsgaard T, Mellgren SI. Outdoor activities and diet in childhood and adolescence relate to MS risk above the Arctic Circle. J Neurol. 2007;254:471-7.

13. van der Mei IAF, Ponsonby A-L, Dwyer T, et al. Past exposure to sun, skin phenotype, and risk of multiple sclerosis: a casecontrol study. BMJ. 2003;327:316-22.

14. Hayes CE, Cantorna MT, Deluca HF. Vitamin D and multiple sclerosis. Proc Sci Exp Biol Med. 1997;216:21-7.

15. Hutter CD, Laing P. Multiple sclerosis: sunlight, diet, immunology and aetiology. Med Hypotheses. 1996;4:67-74.

16. Kripke ML. Ultraviolet radiation and immunology: something new under the sun. Cancer Res. 1995;54:838-43.

17. Axelson O, Landtblom AM, Flodin U. Multiple sclerosis and ionizing radiation. Neuroepidemiology. 2001;20:176-8.

18. McMichael AJ, Hall AJ. Does immunosuppressive ultraviolet radiation explain the latitude gradient for multiple sclerosis? Epidemiology. 1997;8:642-5. 